\title{
Corresponding Authors by chapters
}

1.1

Dr. Orlo H. Clark, MD

UCSF/Mt. Zion Medical Center

Department of Surgery

1600 Divisadero St., Box 1674

Hellman Building, Room C-347

San Francisco 94143-1674 CA, USA

E-Mail clarko@surgery.ucsf.edu

1.2/7.2/7.10/8.9/8.10

Prof. Claudio R. Cernea

Department of Head and Neck Surgery

Univ. of São Paulo Medical School

Alameda Franca, 267-cj 21

Jd. Paulista

01422-000 São Paulo, SP, Brazil

E-Mail cerneamd@uol.com.br

1.3

Dr. Gregory W. Randolph, MD

243 Charles Street

Boston 02114 MA, USA

E-Mail

Gregory_Randolph@meei.harvard.edu

1.4/1.6

Prof. Ashok R. Shaha

Head and Neck Service

Memorial Sloan-Kettering Cancer Ctr.

Cornell Univ. Med. Center

1275 York Ave.

New York 10021 N.Y., USA

E-Mail shahaa@mskcc.org

1.5

Prof. Jean-François Henry, MD

University Hospital Marseille

Department of Endocrine Surgery

264, rue Saint-Pierre

Marseille 13385 Cedex 05, France

E-Mail

jean-francois.henry@mail.ap-hm.fr

1.7

Dr. Jeremy L. Freeman, MD, FRCSC, FACS

Mount Sinai Hospital

600 University Avenue 401

Toronto M5G 1X5, Canada

E-Mail jfreeman@mtsinai.on.ca

1.8

Dr. Erivelto M. Volpi, MD

R. das Figueiras, 551

09080-370 Santo Andre, SP, Brazil

E-Mail eriveltovolpi@hotmail.com
1.9

Dr. William B. Inabnet, MD, FACS

Chief, Section of Endocrine Surgery

Co-Director of New York Thyroid Center

Associate Professor of Clin. Surgery

College of Physicians and

Surgeons of Columbia University

161 Fort Washington Ave.

New York 10032 NY, USA

E-Mailwbi2102@columbia.edu

1.10

Prof. Keith S. Heller, MD, FACS

Professor and Chief of Endocrine Surgery

New York University, School of Medicine

530 First Avuene, Suite $6 \mathrm{H}$

New York 10016 NY, USA

E-Mail keith.heller@med.nyu.edu

1.11

Dr. Fábio Luiz de Menezes Montenegro, MD

Rua Apeninos, 1118 APT 62

Paraiso

04104-021 São Paulo, SP, Brazil

E-Mail fabiomonte@uol.com.br

1.12

Dr. Alfred Simental, MD

Chief Otolaryngology,

Head and Neck Surgery

11234 Anderson St. Suite 2584

Loma Linda 92354 CA, USA

E-Mail asimenta@ahs.llumc.edu

1.13/8.1/8.4

Dr. Dan M. Fliss, MD

Department of Otolaryngology

Head and Neck Surgery

Tel-Aviv Sourasky Medical Center

6 Weizmann St.

Tel Aviv 64239, Israel

E-Mail fliss@tasmc.health.gov.il

1.14

Dr. Marcos R. Tavares, MD

Department of Head and Neck Surgery

University of São Paulo

Medical School

Rua Joaquim Floriano, 101

Conj. 601

04534-010 São Paulo, SP, Brazil

E-Mail Tavares.mr@uol.com.br
1.15

Dr. Patrick Sheahan

125 Pier View Street, No. 109

Daniel Island

Charleston 29492 SC, USA

E-Mail sheahan.patrick@gmail.com

1.16

Prof. Thomas V. McCaffrey, MD, PhD

Professor and Chair

Department of Otolaryngology

Head and Neck Surgery

University of South Florida

12902 Magnolia Drive

Suite 3057

Tampa 33612-9497 FL, USA

E-Mail Thomas.McCaffrey@Moffitt.org

2.1

Dr. Michiel van den Brekel, MD, PhD

Netherlands Cancer Institute

Plesmanlaan 121

1066 CX Amsterdam, The Netherlands

E-Mail m.vd.brekel@nki.nl

2.2

Dr. Yoav P. Talmi, MD, FACS

Chief of Head and Neck Service

The Chaim Sheba Med. Center

Tel Hashomer, Israel 52621

E-Mail yoav.talmi@sheba.health.gov.il

2.3/7.1/7.9/8.5

Prof. Fernando L. Dias, MD, FACS

Chief, Head \& Neck Surgery Dept.

Brazilian National Cancer Institute

Professor of Surgery

Post Graduation School of Medicine

Av. Alexandre Ferreira, 190

Lagoa

2270220 Rio de Janeiro, SP, Brazil

E-Mail fdias@inca.gov.br

2.4

Prof. Francisco Civantos, MD, FACS

Co-Director, Head and Neck Surgery

Associate Professor

Department of Otolaryngology

University of Miami

Sylvester Comprehensive Cancer Ctr.

1475 NW 12 Ave. No. 4027

Miami 33136 FL, USA

E-Mail FCivanto@med.miami.edu 
2.5

Prof. Jesus E. Medina, MD

Department of Otorhinolaryngology

University of Oklahoma

Health Sciences Center

P.O. Box 26901

Oklahoma City 73190 OK, USA

E-Mail jesus-medina@ouhsc.edu

2.6

Dr. John C. O'Brien, Jr., MD

Sammons Cancer Center

Baylor University Medical Center

1004 North Washington Avenue

Dallas 75204-6416 TX, USA

E-Mail job8223@aol.com

2.7

Prof. K. Thomas Robbins, MD

Simmons Cooper Cancer Institute at Southern Illinois University

P.O. Box 19677

Springfield 62794-9677 IL, USA

E-Mail trobbins@siumed.edu

2.8

Dr. Jonas T. Johnson

Dept. of Otolaryngology

University of Pittsburgh

Suite 500, 203 Lothrop Street

Pittsburgh 15213 PA, USA

E-Mail johnsonjt@upmc.edu

\section{9a}

Prof. James Cohen, MD, PhD

Department of Otolaryngology

Head and Neck Surgery

Oregon Health Sci. University

3710 SW US Veterans Hospital Road

Portland 97239 OR, USA

E-Mail James.Cohen2@VA.gov

\section{$2.9 \mathrm{~b} / 7.3$}

Dr. Randal S. Weber, MD, FACS

Department of Head and Neck Surgery University of Texas

M.D. Anderson Cancer Center

1515 Holcombe Boulevard

Box 441

Houston 77030-4009 TX, USA

E-Mail rsweber@mdanderson.org

\subsection{0}

Dr. Rod P. Rezaee, MD, FACS

University Hospital

Case Medical Center

1110 Euclid Avenue

4th Floor Lakeside Bldg.

Cleveland $44106 \mathrm{OH}$, USA

E-Mail rod.rezaee@uhhospitals.org
2.11

Prof. Gary L. Clayman, DMD, MD

Department of Head and Neck Surgery

The University of Texas

M.D. Anderson Cancer Center

1515 Holcombe Boulevard, Unit 441

Houston 77030 TX, USA

E-Mail gclayman@mdanderson.org

2.12/4.10

Dr. Bhuvanesh Singh MD, PhD, FACS

Laboratory of Epithelial Cancer Biology

Head and Neck Service

Memorial Sloan-Kettering Cancer Ctr.

1275 York Avenue

New York 10065 NY, USA

E-Mail singhb@mskcc.org

3.1

Prof. Charles René Leemans, MD, PhD

Professor and Chairman

Department of Otolaryngology

Head and Neck Surgery

VU University Medical Center (VUmc)

P.O. Box 7075

1007 MB Amsterdam, The Netherlands

E-Mailchr.leemans@vumc.nl

3.2

Dr. Neal D. Futran, MD, DMD

Department of Otolaryngology

Head and Neck Surgery

Uni. of Washington School of Medicine

1959 NE Pacific Street, Room BB 1165

Seattle 98195-6515 WA, USA

E-Mail nfutran@u.washington.edu

3.3

Dr. Richard J. Wong, MD

Memorial Sloan-Kettering Cancer Center

Head and Neck Service, C-1069

Department of Surgery

1275 York Avenue

New York 10021 NY, USA

E-Mailwongr@mskcc.org

3.4

Dr. Matthew M. Hanasono, MD

Department of Plastic Surgery

The University of Texas

M.D. Anderson Cancer Center

1515 Holcombe Boulevard, Unit 443

Houston 77030 TX, USA

E-Mailmhanasono@mdanderson.org
3.5

Dr. Jacob Kligerman, MD

Av. Rui Barbosa 870 apto. 901

22250-020 Rio de Janeiro-Flamengo,

SP, Brazil

E-Mail jkligerman@uol.com.br

3.6

Dr. Sheng-Po $\mathrm{HaO}$

14 F, No. 16, Alley 4, Lane 137

Min-Sheng E. Road

Taipei, Taiwan (ROC)

E-Mail shengpo@adm.cgmh.org.tw

4.1

Dr. F. Christopher Holsinger, MD, FACS

Department of Head and Neck Surgery

The University of Texas

M.D. Anderson Cancer Center

1515 Holcombe Boulevard

Box 441

Houston 77030-4009 TX, USA

E-Mail holsinger@mdanderson.org

4.2

Dr. Steven M. Zeitels, MD, FACS

Director

Center for Laryngeal Surgery and

Voice Rehabilitation

Massachusetts General Hospital

One Bowdoin Square

11th floor

Boston 02114 MA, USA

E-Mail steven@mgh.harvard.edu

4.3

Dr. Onivaldo Cervantes

Rua Estela, 515.

Bloco G - cj. 81

04011-002 Viala Mariana

São Paulo, SP, Brazil

E-Mail ocervantes@uol.com.br

4.4/4.9/13.1

Dr. Eugene N. Myers

Distinguished Prof. and Emeritus Chair

Department of Otolaryngology

University of Pittsburgh

School of Medicine

The Eye \& Ear Insitute, Suite 519

200 Lothrop Street

Pittsburgh 15213 PA, USA

E-Mail myersen@msx.upmc.edu

4.5/14.2

Dr. med. Roberto A. Lima, MD

Av. Armando Lombardi, 1000 Bloc2 107

22640-000 Rio de Janeiro, SP, Brazil

E-Mail rlimamd@uol.com.br 
4.6/15.4

Prof. Gregory S. Weinstein, MD, FACS

Professor and Vice Chair

The Department of Otorhinolaryngology

Head and Neck Surgery

The University of Pennsylvania

3400 Spruce Street

Philadelphia 19035 PA, USA

E-Mail

gregory.weinstein@uphs.upenn.edu

\section{7}

Prof. Javier Gavilán, MD

Servicio de ORL

Hospital Unviersitario La Paz

Paseo de la Castellana, 261

28046 Madrid, Spain

E-Mail jgavilan.hulp@salud.madrid.org

4.8/5.2

Prof. Dennis H. Kraus, MD

Memorial Sloan-Kettering Cancer Center

Head and Neck Service

1275 York Avenue

New York 10065 NY, USA

E-Mailkrausd@mskcc.org

\section{1}

Dr. Abrao Rapoport

Head and Neck Surgeon

Hospital Heliopolis

Rua Congeo Xavier, 276 - 10 andar

04231-030 São Paulo, SP, Brazil

E-Mail arapoport@terra.com.br

\section{3}

Dr. Frans JM Hilgers, MD, PhD

Netherlands Cancer Institute

Plesmanlaan 121

1066 CX Amsterdam, The Netherlands

E-Mail f.hilgers@nki.nl

5.4/6.1/6.2/6.3/14.12

Prof. William I. Wei

Li Shu Pui Professor of Surgery

Chair in Otorhinolaryngology

Department of Surgery

University of Hong Kong Med. Ctr.

Queen Mary Hospital

Hong Kong, People's Republic of China

E-Mail hrmswwi@hkucc.hku.hk

\section{4}

Prof. Peter C. Neligan, MB, FRCS

University of Washington Med. Ctr.

Division of Plastic Surgery

1959 NE Pacific St.

Box 356410

Seattle 98195-6410 WA, USA

E-Mail pneligan@uwashington.edu
7.5

Dr. Richard V. Smith, MD, FACS

Department of Otorhinolaryngology

Head and Neck Surgery

3400 Bainbridge Avenue

Bronx 10467 NY, USA

E-Mail rsmith@montefiore.org

7.6

Prof. Bruce J. Davidson, MD, FACS

Professor and Chairman

Department of Otolarynology

Head and Neck Surgery

Georgetown University Medical Center

Washington 20007 DC, USA

E-Mail davidsob@georgetown.edu

7.7

Prof. Alfio José Tincani, MD

Professor of Head \& Neck Surgery

State University of Campinas - UNICAMP

Rua Geraldo Trefiglio 140

13083-793 Campinas, SP, Brazil

E-Mail alfio.jt@gmail.com

7.8

Dr. Randall P. Morton, MB, BS, MSc, FRACS

Counties-Manukau DHB, and

Auckland University

PO Box 98743

South Auckland Mail Centre

Manukau 2240

Auckland, New Zealand

E-Mail rpmorton@middlemore.co.nz

7.11

Dr. Jeffrey D. Spiro, MD

Division of Otolaryngology/

Head and Neck Surgery

University of Connecticut Health Ctr

263 Farmington Avenue MC-6228

Farmington 06030-6228 CT, USA

E-Mailspiro@nso.uchc.edu

7.12

Dr. Kwang Hyun Kim, MD

Department of Otolaryngology

Head and Neck Surgery

Seoul National University

College of Medicine

28, Yeongeon-dong, Jongno-gu

110-744 Korea, South Korea

E-Mail kimkwang@plaza.snu.ac.kr
8.2

Dr. Fernando Walder, MD

Federal University of São Paulo

UNIFESP

Rua Joaquim Floriano, 397/3rd floor

04534-011 São Paulo, SP, Brazil

E-Mail fernandowalder@terra.com.br

8.3

Dr. Eduardo Vellutin

Paça Amadeu Amaral 27/71

01327-010 São Paulo, SP, Brazil

E-Mail evellu@terra.com.br

8.6

Prof. Ehab Hanna MD, FACS

Professor and Vice Chairman

Director of Skull Base Surgery

Medical Director Head and Neck Ctr.

Department of Head and Neck Surgery

University of Texas

M.D. Anderson Cancer Center

1515 Holcombe Boulevard, Unit 441

Houston 77030-4009 TX, USA

E-Mail eyhanna@mdanderson.org

8.7

Dr. Marcos Q.T. Gomes

Praca Amadeu Amaral 27/71

01327-010 São Paulo, SP, Brazil

E-Mailmarcos@dfvneuro.com.br

8.8

Dr. Patrick J. Gullane, MB

Department of Otolaryngology

Head and Neck Surgery

200 Elizabeth Street, 8N-800

Toronto M5G 2C4, Canada

E-Mail Patrick.gullane@uhn.on.ca

9.1/9.2

Prof. James Y. Suen, MD

Professor and Chairman

Department of Otolaryngology

Head and Neck Surgery

4301 W. Markham St.

Little Rock 72205 AR, USA

E-Mail suenjamesy@uams.edu and

suenjamesy@exchange.uams.edu

9.3

Dr. Eduardo Noda Kihara

Hospital Albert Einstein

Neuro Interventional Department

Avenida Albert Einstein, 701

Hemodinamica $4^{\circ}$ andar

05651-091 São Paulo, SP, Brazil

E-Mail kihara@einstein.br 
10.1

Dr. Marcelo D. Durazzo, MD

Praça Amadeu Amaral 47, suite 41 01413-000 São Paulo, SP, Brazil

E-Mail durazzo@attglobal.net

10.2

Dr. Nilton T. Herter, MD

Av. Independência 1211 Sala 201

90035-075 Porto Alegre, SP, Brazil

E-Mailnherter@uol.com.br

11.1

Dr. Nadir Ahmad

Department of Otolaryngology

Head and Neck

Vanderbilt University Medical Center

7209 Medical Center East, South Tower

1215 21st Avenue South

Nashville 37232-8605 TN, USA

E-Mail nadirahmad@hotmail.com

11.2

Dr. Ziv Gil, MD

Department of Otolaryngology

Head and Neck Surgery

Tel-Aviv Sourasky Medical Center

6 Weizmann Street

64239 Tel-Aviv, Israel

E-Mail ziv@baseofskull.org

11.3

Dr. Kerry D. Olsen, MD

Mayo Clinic Rochester

200 First Street Southwest

Rochester 55905 MN, USA

E-Mail olsen.kerry@mayo.edu

12.1

Prof. Flávio C. Hojaij, MD

Rua Padre João Manuel 450, cj 18

01411-001 São Paulo, SP, Brazil

E-Mail fchojaij@uol.com.br

12.2

Dr. Dorival De Carlucci, Jr., MD

Rua Padre João Manuel 45, room 18

Cerqueira César

01411-001 São Paulo, SP, Brazil

E-Mail decarlucci@uol.com.br

13.2

Dr. Carlos N. Lehn, MD

Chief of the Head and Neck Surgery

Service

Hospital Heliópolis, São Paulo

Rua Joaquim Floriano 636 ap 22

04534-002 São Paulo, SP, Brazil

E-Mail cnlehn@terra.com.br
13.3

Prof. David W. Eisele, MD, FACS

Professor and Chairman

400 Parnassus Avenue

Suite A-730

San Francisco 94143-0342 CA, USA

E-Mail deisele@ohns.ucsf.edu

14.1

Dr. Luiz Carlos Ishida, MD

Plastic Surgery Division of the

Faculty of Medicine of the

University of São Paulo

Rua Itamiami, 35

Vila Mariana

04120-100 São Paulo, SP, Brazil

E-Mail Ici@uol.com.br

\section{3}

Dr. José Magrim, MD, PhD

Head and Neck Surgery and Otorhinolaryngology Department

Hospital AC Camargo

Rua Professor Antonio Prudente, 211

01509-900 São Paulo, SP, Brazil

E-Mail jgon13@terra.com.br

14.4

Prof. Richard E. Hayden, MD

Professor and Chair

Department of Otolaryngology

Head and Neck Surgery

5777 East Mayo Boulevard

Phoenix 85054 AZ, USA

E-Mail hayden.richard@mayo.edu

14.5

Prof. Gady Har-El, MD, FACS

Chairman, Dept. of Otolaryngology

Head and Neck Surgery

Lenox Hill Hospital, New York

Prof. of Otolaryngology \& Neurosur.

State University of New York

Downstate Medical Center

Brooklyn 11201 NY, USA

E-Mail gadyh@aol.com

14.6/14.8/14.10

Dr. Julio Morais, MD, PhD

Assistant Professor of Plastic Surgery

São Paulo University Medical School

Rua Baronesa de Bela Vista, 196

04612-000 São Paulo, SP, Brazil

E-Mailjmorais@br2001.com.br
14.7

Dr. Mark L. Urken, MD

Beth Israel Medical Center

10 Union Square East, Suite 5B

New York 10003 NY, USA

E-Mail murken@chpnet.org

14.9

Dr. Mario S.L. Galvao, MD

Reconstructive Microsurgery Unit

National Cancer Institute

Rua Visconde Silva 52/suite 1006

Botafogo, Rio de Janeiro, SP, Brazil

E-Mail galvaorj@iis.com.br

14.11

Prof. John J. Coleman, 3rd, MD

Professor of Surgery

Chief of Plastic Surgery

Indiana University School of Med.

Roudebush VAMC- Indianapolis

Indianapolis 46204 IN, USA

E-Mail jjcolema@iupui.edu

15.1

Dr. Paulo Campos Carneiro, MD, PhD

University of São Paulo

School of Medicine

Department of Pathology

Av. Rebouças 353 cj. 114

05401-000 Cerqueira Cesar,

São Paulo, SP, Brazil

E-Mail p.carneiro@saudetotal.com.br

15.2

Dr. Pedro Michaluart, Jr., MD

Head and Neck Service

Hospital das Clinicas da Faculdade

de Medicina da Universidade

de São Paulo

R. Dr. Enéas de Carvalho Aguiar, 255

No. 8 andar, Sala 8074

05403900 São Paulo, SP, Brazil

E-Mail pemic@uol.com.br

15.3

Dr. Erich M. Sturgis, MD, MPH

Department of Head and Neck Surgery

and Epidemiology

The University of Texas

M.D. Anderson Cancer Center

1515 Holcombe Boulevard, Unit 441

Houston 77030-4009 TX, USA

E-Mail esturgis@mdanderson.org 